\title{
Prise En Charge Des Anévrismes Artériels Dans Un Centre Africain Non Spécialisée
}

\author{
James Didier L., \\ Abdoulaye MB.,
}

Département de Chirurgie et Spécialités Chirurgicales,

Hôpital National de Niamey.

Bako H.,

Département de Cardiologie, Exploration Cardiovasculaire,

Hôpital National de Niamey.

\author{
Ide $K$. , \\ Saidou A., \\ Ide $G$., \\ Hama Y., \\ Sani Rabiou,
}

Département de Chirurgie et Spécialités Chirurgicales,

Hôpital National de Niamey.

Chaibou MS,

Daddy $\mathrm{H}$.,

Département d'anesthésie, de Réanimation et des Urgences,

Hôpital National de Niamey.

\section{Adakal O.,}

Faculté des Sciences de la Santé de l'Université de Maradi /Niger

Sidibe T.,

Service de Radiologie à l'hôpital national de Niamey, Niger

\section{Ouaissi M.,}

Service de Chirurgie Digestive, Oncologique, Endocrinienne Et Transplantation Hépatique, Tours, France.

\section{Harouna Y.,}

Département de Chirurgie Et Spécialités Chirurgicales,

Hôpital National de Niamey.

\section{Abarchi H.,}

Département de Chirurgie et Spécialités Chirurgicales,

Hôpital National de Lamorde, Niamey

Sani R.,

Département de Chirurgie et Spécialités Chirurgicales, Hôpital National de Niamey. 


\section{Abstract}

Introduction: Arterial aneurysms affect 7 to $8 \%$ of people over 65 in the West and are the 2nd leading cause of death in these countries. In Africa this frequency is poorly evaluated. The objective of this work is to report the management of arterial aneurysms at the National Hospital of Niamey (HNN). Patients and methods: This was a retrospective, descriptive study over a period of eight (8) years from January 2009 to December 2016, performed in the surgical departments of the National Hospital of Niamey. Included in the study were patients of both sexes, hospitalized and / or operated for arterial aneurysm. Not included were patients treated for arterial aneurysm with incomplete records or those concerning the neurosurgical sphere. Results: During the study period, 17,748 patients were hospitalized in the general surgery departments, including 16 patients for arterial aneurysm, or $0.09 \%$ of surgical pathologies. There were 13 men (81.25\%) and 3 women (18.75\%), or a sex ratio of 4.33 . The average age was 55.75 years with extremes ranging from 25 years old to 90 years old. The circumstances of discovery of the aneurysms were swelling of the antero-internal aspect of the thigh in 7 cases $(43.75 \%)$, abdominal mass 6 cases $(37.50 \%)$, then 2 cases $(12.50 \%)$ of chest pain and incidental discovery in 1 cas $(6.25 \%)$. The most common risk factor was high blood pressure with $43.75 \%$ ( 7 cases). The aneurysm sat on the femoral artery in $43.75 \%(n=7)$, of which 6 on the deep femoral and 1 on the superficial femoral, on the infrarenal aorta in $31.25 \%(\mathrm{n}=5)$, on thoracic aorta $12.50 \%(n=2)$, on the iliac artery $12.50 \%(n=2)$. For the diagnosis the angioscanner is realized in all the patients and in addition Doppler ultrasound in $43,75 \%$ of cases. Twelve (12) patients benefited from curative surgical intervention by prosthetic graft by PTFE in $58.33 \%$ of cases and by Dacron in $41.66 \%$. The average stay was 29.75 days and the immediate operative followup was complicated by thrombosis in $12.5 \%$ and parietal suppurations in $6.25 \%$. We recorded two (2) deaths, ie $12.5 \%$ among non-operated patients. Conclusion: Arterial aneurysms are rare diseases at the HNN. Conventional surgery is the treatment performed in our patients. The postoperative course was simple in most cases.

Keywords: Aneurysm, arterial, prosthesis, classical surgery

\section{Resume}

Introduction : Les anévrismes artériels affectent 7 à $8 \%$ de personnes âgées de plus de 65 ans en occident et constituent la 12ème cause de décès dans ces pays. En Afrique cette fréquence est mal évaluée. L’objectif de ce travail est de rapporter la prise en charge des des anévrismes artériels à 
l'Hôpital National de Niamey (HNN). Patients et méthodes : Il s'agissait d'une étude rétrospective, descriptive sur une période de huit(8) ans allant de janvier 2009 à décembre 2016, effectuée dans les services de chirurgie de l'hôpital National de Niamey. Etaient inclus dans l'étude, les patients des deux sexes, hospitalisés et/ou opérés pour anévrisme artériel. N’étaient pas inclus, les patients pris en charge pour anévrisme artériel dont les dossiers sont incomplets ou ceux concernant la sphère neurochirurgicale. Résultats : Durant la période d'étude ,17 748 patients ont été hospitalisés dans les services de chirurgie générale dont 16 patients pour anévrysme artériel soit $0,09 \%$ des pathologies chirurgicales. Il s'agissait de 13 hommes $(81,25 \%)$ et de 3 femmes $(18,75 \%)$, Soit un sex ratio de 4,33. La moyenne d'âge était de 55,75 ans avec des extrêmes allant de 25 ans et 90 ans. Les circonstances de découverte des anévrismes étaient une tuméfaction de la face antero-interne de la cuisse dans 7 cas $(43,75 \%)$, une masse abdominale 6 cas $(37,50 \%)$, puis 2 cas $(12,50 \%)$ de douleur thoracique et de découverte fortuite dans 1cas $(6,25 \%)$. Le facteur de risque le plus retrouvé était l'hypertension artérielle avec $43,75 \%$ ( 7 cas). L'anévrysme siégeait sur l'artère fémorale dans $43,75 \%(n=7)$ dont 6 sur la fémorale profonde et 1 sur la fémorale superficielle, sur l'aorte sous rénale dans $31,25 \%(n=5)$, sur de l'aorte thoracique $12,50 \%(n=2)$, sur l'artère iliaque $12,50 \%(\mathrm{n}=2)$. Pour le diagnostic l'angioscanner est réalisé chez tous les patients et en plus l'échographie doppler dans $43,75 \%$ de cas. Douze(12) patients ont bénéficié d'une intervention chirurgicale curative par mise à plat greffe prothétique par PTFE dans 58,33\% de cas et par Dacron dans $41,66 \%$. Le séjour moyen était de 29,75 jours et les suites opératoires immédiates étaient compliquées de thrombose dans $12,5 \%$ et suppurations pariétales dans 6,25\%.Nous avions enregistré deux(2) décès soit 12,5\% parmi les patients non opérés. Conclusion: Les anévrismes artériels sont des pathologies rares à l'HNN. La chirurgie conventionnelle est le traitement réalisé chez nos patients. Les suites opératoires étaient simples dans la plupart des cas.

Mots-clés : Anévrisme, artériel, prothèse, chirurgie classique

\section{Introduction}

L'anévrysme artériel est inégalement réparti dans le monde. Il affecte 7 à $8 \%$ de personnes âgées de plus de 65 ans en occident et constitue la 12 ème cause de décès dans ces pays, soit environ 12000 à 15000 décès annue1 (Ricco, 2011 ; Déglise, 2013).En Afrique subsaharienne l'anévrisme artériel est peu fréquent, sans doute mal évalué, car la situation est particulièrement caractérisée par une rareté des chirurgiens vasculaire et une insuffisance du plateau technique. Ceci pourrait en partie expliquer le nombre faible des cas pris en charge. Ces dernières années on note cependant une augmentation de 
l'incidence des anévrismes du fait, du vieillissement de la population et de l'augmentation des facteurs de risques cardio-vasculaires (Sule, 2012). En I990 en Afrique du Sud 30 cas ont été colligés sur une période de 16 ans (Louridas ,1990). Au Mali, en 2009 il a été rapporté 7 cas en 2 ans (Beye, 2009). La complication la plus redoutée est la rupture qui est le plus souvent fatale. Tous les territoires vasculaires peuvent être concernés et les anévrismes sont parfois multiples. L'anévrisme de l'aorte abdominale sous rénale (AAASR) est l'anévrisme artériel le plus fréquent $90 \%$ [6] suivi des anévrismes poplités qui constituent $70 \%$ des anévrismes périphériques (Baleato, 2008; Duffy, 1998).Le traitement se fait par la mise en place d'une prothèse par chirurgie conventionnelle ou endovasculaire. Cependant on peut avoir recours au greffon veineux. Ainsi la difficulté d'avoir à la fois d'avoir un plateau technique suffisant et les chirurgiens ayant une connaissance diagnostique et thérapeutique de leur prise en charge, sont souvent responsables d'un haut taux de mortalité. Le Niger ne présente pas à ce jour de trauma center ou de centre spécialisé en chirurgie vasculaire. Au sein de notre institution nous avons mis en place un programme d'enseignement nous permettant d'appréhender au mieux la pathologie anévrismale dans un environnement de chirurgie générale non spécialisée. Notre plateau technique ne permet de réaliser des traitements endovasculaires. Nous rapportons notre prise en charge sur les 8 dernières années d'une pathologie rare dans nos contrées, associés à un enseignement vasculaire adapté à notre plateau technique.

\section{Patients et méthodes :}

Entre 01/01/2009 et 31/12/2016, 17748 patients ont été pris en charge au sein de notre département. 16 patients consécutifs ont consulté pour une pathologie anévrismale. Seuls les anévrismes des artères digestives ou des membres ont été inclus, les anévrismes artériels cérébraux ont été exclus. Les données cliniques préopératoires, Les circonstances de découverte, Les facteurs de risque, Les caractéristiques de l'anévrisme (diamètre et aspect), le siège antécédent de diabète, dénutrition préopératoire) et de la chirurgie (type d'intervention, type de chirurgie vasculaire) ont été colligée de manière rétrospective.

La pathologie anévrismale est peu connue dans nos pratiques, car très peu rencontrée. Ceci fait qu'on n'y pense pas même devant des signes d'appels. Pour améliorer la prise en charge de cette pathologie, en l'absence d'un service de chirurgie vasculaire, c'est le service de chirurgie générale qui prend en charge ces patients surtout en urgence. Ceci dans le but non seulement de sauver ces patients mais aussi l'occasion pour les apprenants de revoir la sémiologie de cette pathologie, afin d'y penser devants des signes d'appel et de mieux les orienter et les prendre en charge. Les complications postopératoires ont été évaluées en utilisant la classification de Clavien-Dindo. 
Le suivi clinique et biologique post opératoire réalisait à 6 mois incluait les données cliniques et paracliniques. A la sortie les patients opérés sont sous acide acétyle salicylique $160 \mathrm{mg} / \mathrm{J}$. Ce traitement à base d'antiagrégant plaquettaire est maintenu à vie pour les patients qui ont un facteur de risque cardiovasculaire. Il est arrêté au bout de deux ans pour les patients qui n'ont aucun facteur de risque.

\section{Résultats :}

Durant la période d'étude ,17 748 patients ont été hospitalisés dans les services chirurgie générale dont 16 patients pour anévrysme artériel soit $0,09 \%$ des pathologies chirurgicales. Il y avait 13 hommes $(81,25 \%)$ et 3 femmes $(18,75 \%)$. L'âge moyen est de 55,75 ans $[ \pm 15,32]$ avec des extrêmes de 38 ans et 90 ans. (25-90) ans. Set patients (43,75\%) avaient une HTA. Les modalités de découverte étaient une douleur thoracique dans $12,50 \%(\mathrm{n}=2)$, une tuméfaction de la face antéro-interne de la cuisse dans $43,75 \% \quad(\mathrm{n}=7)$, une masse abdominale 37,50\% ( $\mathrm{n}=6)$.

Un angiscanner a été réalisé chez les patients (100\% des cas) associé dans $43,75 \%(\mathrm{n}=7)$ a une échographie doppler.

L'anévrysme siégeait sur l'artère fémorale dans $43,75 \%(\mathrm{n}=7)$ dont 6 sur la fémorale profonde et 1 sur la fémorale superficielle, sur l'aorte sous rénale dans $31,25 \%(\mathrm{n}=5)$, sur de l'aorte thoracique $12,50 \%(\mathrm{n}=2)$ dont 1 sur la portion ascendante et 1 sur les portions horizontales et descendante, sur l'artère iliaque $12,50 \%(\mathrm{n}=2)$ dont 1 sur l'iliaque externe gauche et 1 sur l'iliaque commune gauche. (Figure 2).

Le diamètre médian était de $6,4 \mathrm{~cm}$ sur $8,9 \mathrm{~cm}$ avec des extrêmes de $4,2 \mathrm{~cm}$ sur.25 cm. Soixante-quinze pourcent $(75 \%)$ des anévrismes était supérieur à $5 \mathrm{~cm}$...

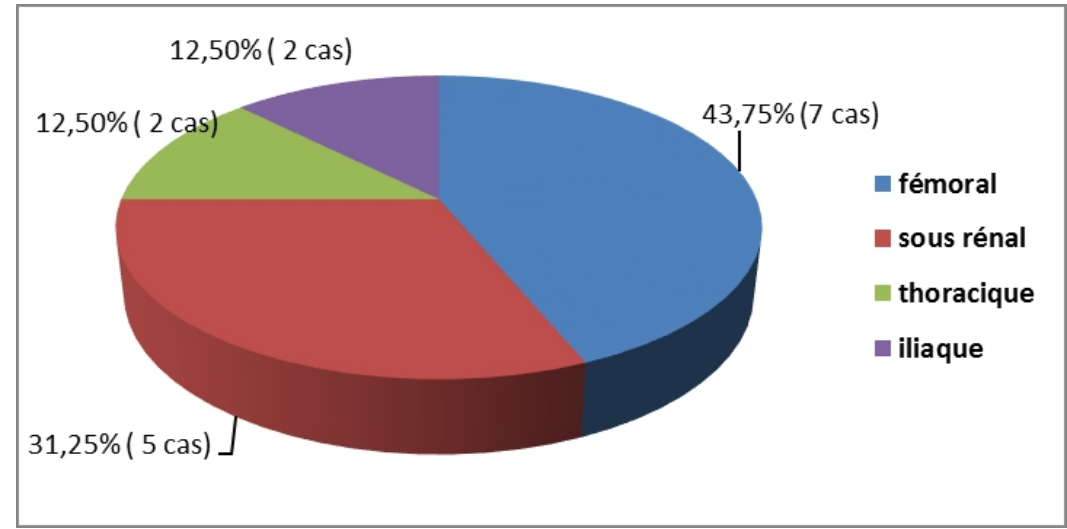

Figure 2 : Répartition des patients selon le siège de l'anévrysme 


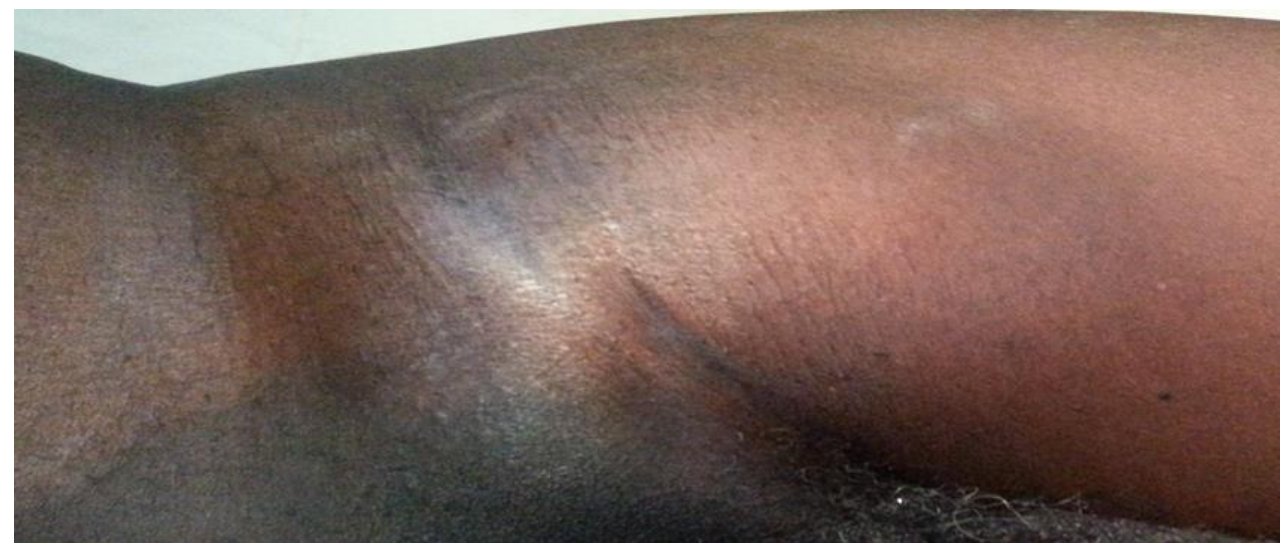

Image 1 : anévrysme de l'artère fémorale profonde

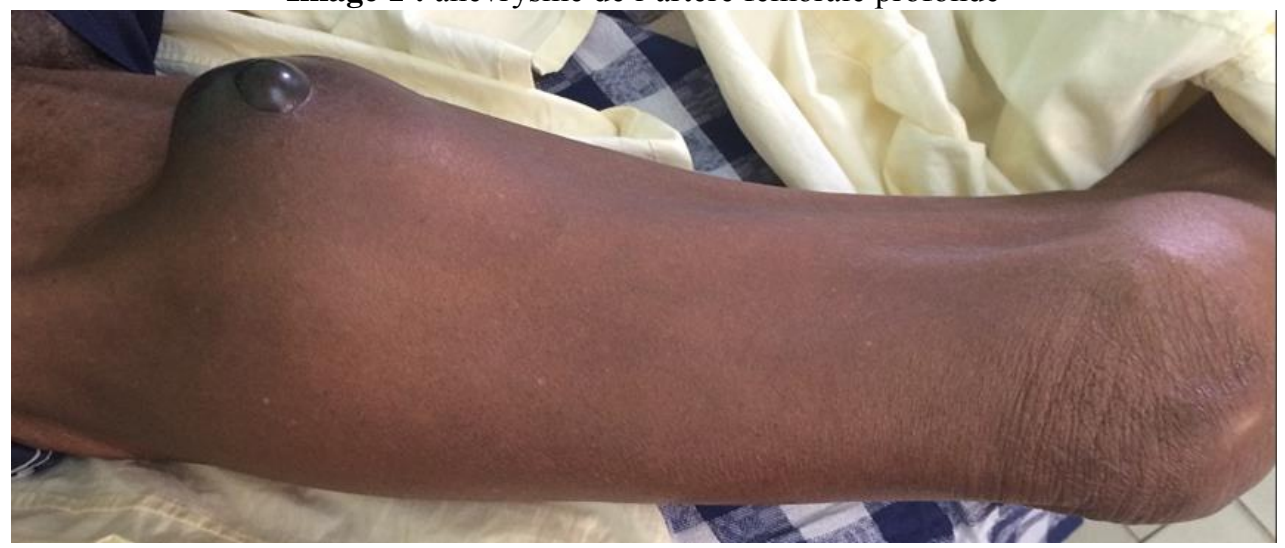

Image 2 : anévrysme de l'artère fémorale superficielle

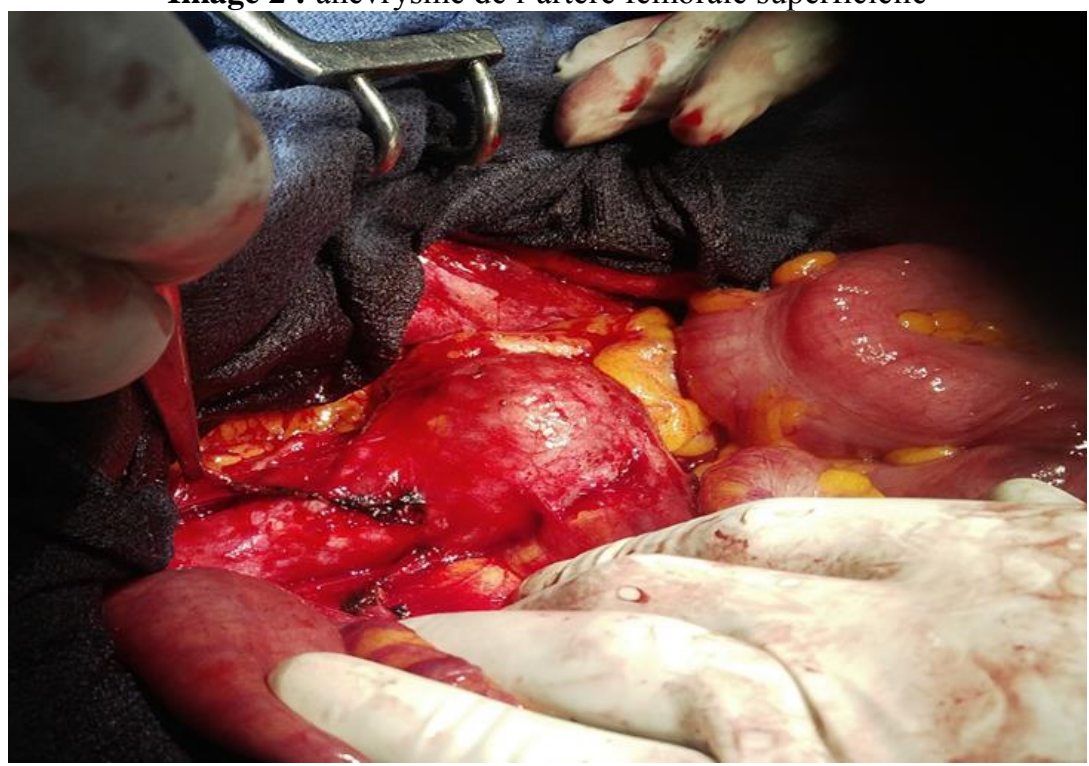

Image 3 : anévrysme de l'aorte sous rénale 


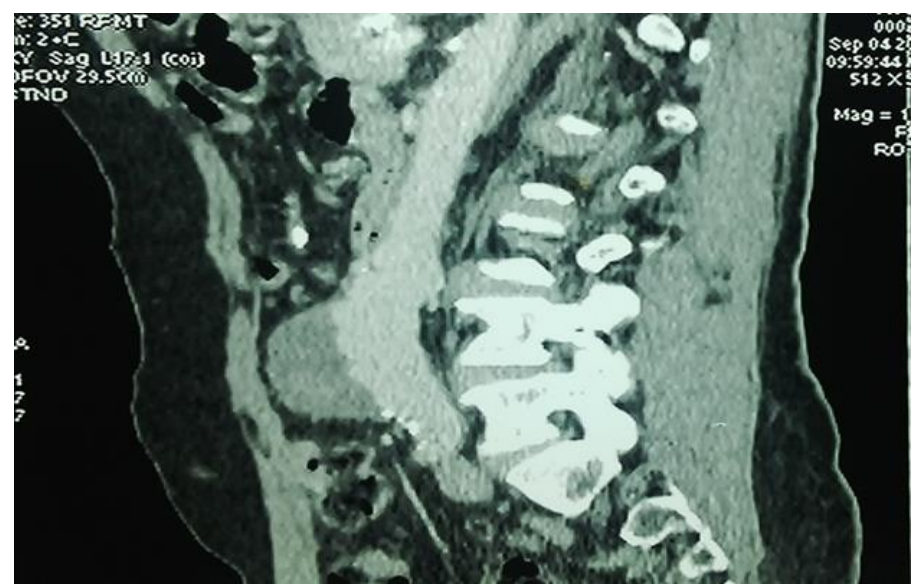

Image 4 : anévrysme de l'aorte sous rénale (angioscanner)

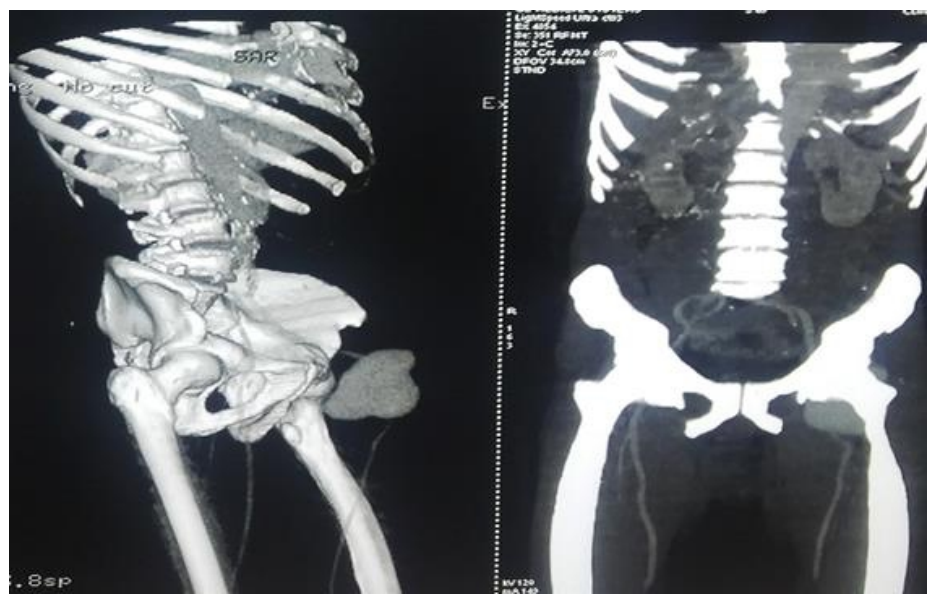

Image 5 : anévrysme de l'artère fémorale (angioscanner)

Quatre patients n'ont pu être opéré par la nondisponibilité d'une CEC et une impossibilité de les transporté, et pour 2 autres il n'y avait pas de prothèse.

Pour les 12 autres patients la chirurgie conventionnelle tait utilisées...

Sur le plan thérapeutique la chirurgie conventionnelle était la seule technique utilisée. Dans le cadre de la préparation à l'acte opératoire tous les patients ont reçu de l'héparine à dose préventive (lovenox 0,40 par $\mathrm{j}$ ou Héparine non fractionnée $100 \mathrm{ui} / \mathrm{kg} / \mathrm{J}$ ) deux heures avant l'acte opératoire. Tous les patients ont été opérés sous anesthésie générale. Sur les seize patients, $75 \%(\mathrm{n}=12)$ avaient été opérés. Pour les 4 patients non opérés les raisons étaient dans 2 cas des anévrysmes de l'aorte thoracique par manque de dispositif de circulation extracorporelle nécessaire pour ce type d'intervention ; pour les 2 autres cas il n'y avait pas de prothèses appropriées (calibres). Deux types de prothèse avaient été utilisés. Il s'agissait du polytetrafluoroethylene(PTFE) 
dans 7 cas soit 58,33\% des patients opérés et le polyester (dacron) dans 5 cas soit 41,66 \%. Parmi les douze (12) anévrismes opérés, 7 étaient fémoraux soit $58,33 \%$, trois (3) AAASR soit $25 \%$ et deux (2) anévrismes iliaques soit 16,66 $\%$. (Figure 3).

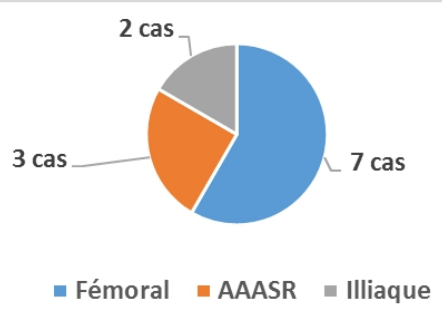

Figure 3 : Répartition des patients selon le siège des anévrismes opérés :

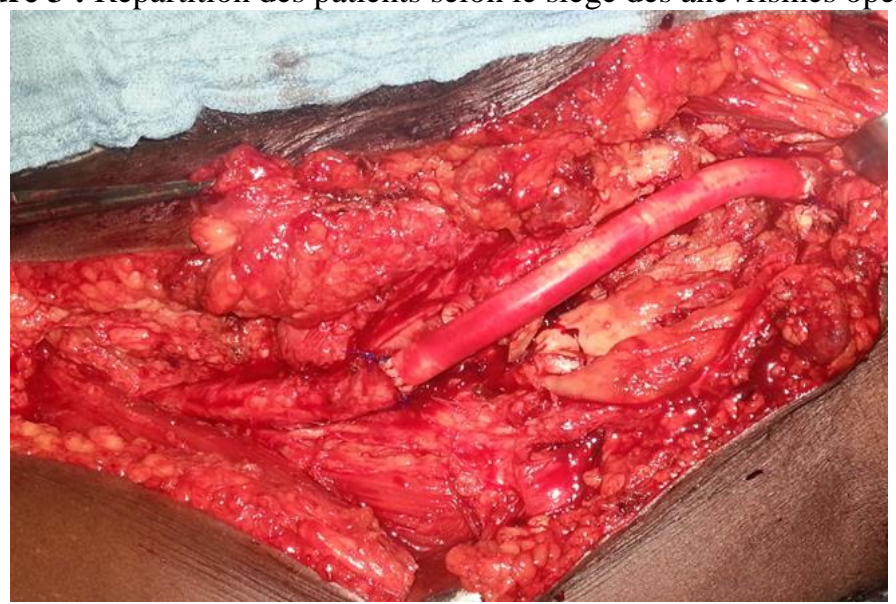

Image 6 : mise en place de la prothèse PTFE

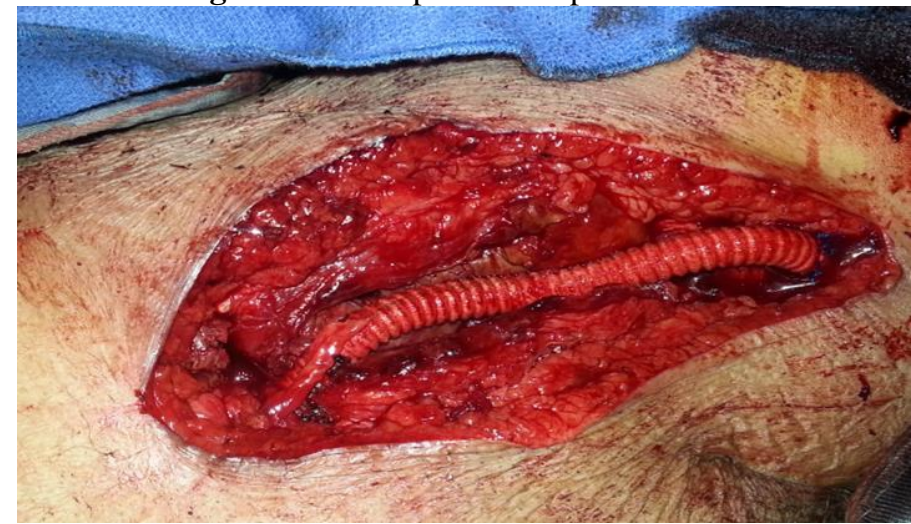

Image 7 : mise en place de la prothèseen Dacron

Aucune transfusion en peropératoire n'a été faite. Au moment de la pose de la prothèse, une dose de charge d'héparine non fractionnée de $300 \mathrm{UI} / \mathrm{kg}$ est administrée. En post opératoire tous les patients ont reçu de l'enoxaparine à dose curative (lovenox $0,6 \mathrm{ml}$ deux fois par jour) jusqu'à leur sortie de l'hôpital. 
Le séjour moyen était de 29,75 jours. Les suites opératoires étaient simples pour $75 \%$ des patients $(\mathrm{n}=9)$ et compliquées dans 3 cas $(25 \%)$.

Les complications postopératoires étaient une thrombose dans 2 cas et une suppuration pariétale dans 1 cas (Tableau I). La thrombose intéressait un cas d'anévrysme de l'artère fémorale traité par embolectomie par une sonde de fogarty. Pour l'autre patient la veine fémorale thrombosée a été traitée par un anticoagulant à dose curative. La perméabilité de la veine fémorale a été obtenue au bout de 4 jours. Le cas de suppurations pariétales était survenu sur un cas d'anévrysme de l'artère fémorale gauche. Le patient été réadmis et une antibiothérapie adaptée à l'antibiogramme a été instituée, avec pansement biquotidien ; l'état du patient s'est amélioré avec tarissement de la suppuration. Les suites opératoires des AAASR étaient simples. Aucune mortalité n'a été recensée parmi les patients opérés. Par contre il y avait deux(2) décès parmi les patients non opérés (les 2 AAASR) probablement par rupture d'anévrisme.

Tableau I : récapitulatif des complications :

\begin{tabular}{lll}
\hline Patients & Mortalité & Morbidité \\
\hline Patients opérés : 12 $(\mathbf{7 5 \%})$ & $\mathbf{0}$ & $\mathbf{3 ( 1 8 , 7 5 \% )}$ \\
Patients non opérés : 4 (25\%) & $\mathbf{2 ( 1 2 , 5 \% )}$ & \\
\hline Total & $\mathbf{2 ( 1 2 , 5 \% )}$ & $\mathbf{3 ( 1 8 , 7 5 \% )}$ \\
\hline
\end{tabular}

Le suivi à 3, 6 mois et 2 ans était satisfaisant pour les 12patients opérés.

\section{Discussion :}

Dans cette étude portant sur les anévrismes artériels à l'HNN, sur une période de 8 ans, 16 patients ont été recensés, soit de 0,09\% des pathologies chirurgicales. Cette faible fréquence est rapportée dans la littérature variant de 0,01 à $0,2 \%$ (Raad, 2007).L'âge moyen des patients est de 55,75 ans avec des extrêmes de 25 ans à 90 ans. La survenue possible des anévrismes à un âge jeune est donc possible. Dans d'autres séries l'âge moyen variait de 40,72 ans à 60 ans avec des extrêmes allant de 30 à70 ans. (Schneider, 2011 ; Lapari, 2006 ; Muscari, 2002 ; Raherinantenaina, 2015), Dans cette série il y’a une prédominance masculine avec $81,25 \%$ d'hommes pour $18,75 \%$ de femmes. Cette prédominance masculine est rapportée par plusieurs auteurs (Muscari, 2002 ; Raherinantenaina, 2015 ; Puppinck, 2004). Les circonstances de découverte l'anévrisme est une tuméfaction de la face antéro-interne de la cuisse dans la majorité des cas dans cette série, suivi de masse abdominale, de douleur thoracique et dans peu de cas découverte fortuite. Ces circonstances sont similaires à celles de plusieurs autres séries (Raherinantenaina, 2015 ; Lyazidi, 2015).Néanmoins certains auteurs ont rapporté la découverte l'anévrisme au stade de rupture (Belmir , 2011).

Les facteurs de risque cardiovasculaire retrouvés dans cette série sont l'hypertension artérielle et le Tabagisme. Ces facteurs sont parmi les 
principaux facteurs de risques cardiovasculaires décrits dans la littérature ainsi que l'éthylisme qui est souvent associé (Lyazidi, 2015 ; Quandale, 1998).

Le diamètre moyen retrouvé est de $6,4 \mathrm{~cm}$ sur $8,9 \mathrm{~cm}$, avec des extrêmes de $4,2 \mathrm{~cm}$ sur $25 \mathrm{~cm}$ ce qui dénotait la découverte tardive des anévrismes, le plus souvent au stade symptomatique. Cette découverte tardive avec des diamètres parfois importants de l'anévrysme allant de $8 \mathrm{~cm}$ à $11 \mathrm{~cm}$ est retrouvée dans plusieurs séries, avec chez certains patients plusieurs localisations anévrysmales (Raherinantenaina, 2015 ; Rezzeki, 2015 ; Salomon, 2016). Pour la forme, il a été retrouvé autant d'anévrysmes sacciformes que fusiformes..

Ces deux formes des anévrysmes sont les plus connues même si dans certaines publications il n'est rapporté que la forme sacciforme (Lyazidi, 2015 ; Tebere, 2013 ; Hiroshi, 2010). Le siège de l'anévrysme le plus rencontrés est fémoral, suivi de celui de l'aorte abdominale,. Ceci rejoint les données de la littérature dans lesquelles le siège fémorale est le plus retrouvés même si la plupart des études portent souvent un cas (Raad, 2007 ; Raherinantenaina, 2015 ; Hiroshi, 2010 ; Mleyhi, 2013).

L'angioscanner a été réalisée chez tous les patients, parfois associé à l'échographie doppler dans la plupart des cas. Ces deux examens font le diagnostic et permettent d'apprécier la gravité des lésions facilitant ainsi la décision thérapeutique avec une efficacité sur la prise en charge. Dans la plupart des études l'angioscanner a été effectué pour tous les patients [14,22]. Par contre dans d'autres séries, cet examen n'a été réalisé que dans deux tiers des cas (Lapari, 2006).

Dans cette étude parmi les 16 patients, 12 patients sont opérés, et tous ces patients ont bénéficié d'une chirurgie conventionnelle avec mise à platgreffe de prothèse. Les autres (4patients) n'ont pas pu être opérés par abstention opératoire comme rapporter par plusieurs auteurs où la chirurgie à ciel ouvert a été utilisée (Lyazidi, 2015; Salomon, 2016 ; Hiroshi, 2010). Par contre Raherinantenaina $\mathrm{F}$ a effectué à la fois, la chirurgie conventionnelle dans 56\% de cas et la chirurgie endovasculaire dans 36\% (Raherinantenaina, 2015). Malgré l'essor des endoprothèses avec la robotique et la navigation endovasculaire avec le navigateur Magellan ou Hansen, la chirurgie conventionnelle garde une place prépondérante dans la chirurgie vasculaire.

Sept(7) patients ont bénéficié de prothèse en PTFE et cinq(5) patients de prothèse en Dacron contrairement à d'autres études où les auteurs n'ont utilisé que la prothèse PTFE seule ou de dacron également seule pour tous les patients (Lyazidi, 2015; Salomon, 2016 ; Hiroshi, 2010).Le choix entre la prothèse en Dacron et celle en PTFE dépend de la disponibilité et des habitudes du chirurgien. Le pontage par greffon veineux autologue utilisant la veine saphène ou même de greffon artériel utilisant l'artère fémorale superficielle reste également une alternative possible. Dans cette sérié, la durée de séjour moyen est de 29,75jours.Le séjour hospitalier varie selon les séries ; dans 
d'autres ce séjour est court (7jours) (Schneider, 2011 ;Rezzeki, 2015), parfois plus ou moins long allant de 13 à 24 jours (Lapari, 2006 ; Hiroshi, 2010). Parmi les 12 patients opérés les suites opératoires sont simples dans 9 cas (75\%) et compliquées dans 3 cas $(25 \%)$.dont deux(2) cas de thromboses et 1 cas de suppuration pariétale. Des complications sont également rapportées par certains auteurs de l'ordre de 5\% (Raherinantenaina, 2015) .par contre dans d'autres publications aucune complication n'a été rapportée (Lapari, 2006).

\section{Conclusion}

Les anévrismes artériels sont des pathologies rares dans notre pratique. Ces anévrismes étaient habituellement retrouvés chez les sujets âgés majoritairement de sexe masculin. Le fait inattendu était la survenue chez les jeunes. Les localisations des anévrismes étaient fémorale, aortique, iliaque. La chirurgie conventionnelle est le traitement réalisé. Les suites opératoires étaient simples dans la plupart de cas. Le diagnostic des anévrismes est aisé mais le manque de matériels rend la prise en charge assez difficile dans nos hôpitaux.

\section{References:}

1. Ricco JB, Régnault de la Mothe G. Chirurgie des anévrysmes artériels des membres. Encycl Méd Chir, Techniques chirurgicales - Chirurgie vasculaire 43-028-A. 2011;21p.

2. Déglise $S$, Dubuis $C$, François $S$ et al. Prise en charge diagnostique et thérapeutique des anévrismes de l'aorte thoracique et/ou abdominale.Forum Med Suisse. 2013 ;13(37) :719-724.

3. Sule A, Ardil B, Ojo E. Abdominal aortic aneurysm and the challenges of management in a developing country: A review of three cases. Ann Afr Med .2012; 11( 3): 176-181.

4. Louridas G, Reilly K, Perry MO. The role of the abdominal aortic aneurysm diameter measurement to computed tomography. South Afr Med J. Johannesburg. 1990; 78(11) :642-3.

5. Beye SA, Kane O, Tchikangoua TN. Anesthésie et réanimation pour chirurgie d'anévrisme de l'aorte abdominale sous-rénale. Mali Méd. $2009 ; 24(1): 9-12$.

6. Baleato S, Bierry G, Garcia-Figueiras R. Anévrismes artériels ; revue des différents territoires. Journées françaises de radiologie paris. 2008 oct : 24-28.

7. Duffy ST, Colgan MP, Sultan S et al. Popliteal aneurysms: a 10-year experience. Eur J Vasc Endovasc Surg. 1998;16: 218-222.

8. Raad E, Demaria R, Rouvière $\mathrm{P}$ et al. Les anévrismes des artères digestives. À propos d'un cas clinique de localisation anévrismale multiple et revue de la littérature. J Mal Vasc.2007;32(4-5) :216-220. 
9. Schneider F, Kobeiter H, Allaire E et al. Traitement endovasculaire des anévrismes non compliqués de l'aorte abdominale chez le sujet agé. J Mal Vasc. $2011 ; 36(2): 94-94$.

10. Lapari G, MigliaraB ,Cavalina A et al. Traitement des anévrismes du tronc cœliaque : expérience personnelle et revue de la littérature. J Mal Vasc. 2006 ;31(2) :72-75.

11. Muscari F ,Barret A, Chaufour x et al . Prise en charge des anévrismes des artères digestives : étude rétrospective de 23 cas. Ann chig. 2002 ; $127: 281-8$.

12. Raherinantenaina F, RajaonanaharyTMA ,Rakoto R. Les faux anévrismes de 1'artère fémorale en orthopédie et en traumatologie.J Mal Vasc. $2015 ; 40(6): 376-383$.

13. Puppinck P, Chevalier J, Ducasse E, Dasnoy D. L'autotransplantation de l'artère fémorale superficielle dans les lésions artérielles poplitées. Une bonne alternative?.J Mal Vasc. 2004;29(1):9-11.

14. Lyazidi Y, AbissegueY,Chtata HT et al . Traitement endovasculaire d'un anévrisme de l'artère sous Clavière gauche intra-thoracique. J Mal Vasc. $2015 ; 40(4): 265-269$.

15. Belmir H, Azghari A, Mechchat A et al. Rupture d'un faux anevrisme de 1 artére poplitée révelant une exostose du tibia a propos d'un cas et revue de la literature.J Mal Vasc.2011;36(1):50-55.

16. Quandale P, Gambiez L, Brami F et al. Hémorragie digestive par rupture d'un anévrisme des artères viscérales. Présentation de quatre observations. Ann chirg.1998;123(2) :139-47.

17. Rezzeki A, Ouledtaib A, Elhoumaidi A et al . Faux anévrisme de l'artère iliaque interne au cours de la maladie de Behcet. J Mal Vasc. 2015 ;40(3) :196-199.

18. Salomon du mont L ,Holzer T, Kazandjian C et al . Un anévrisme isolé de l'artère fémorale profonde. J Mal Vasc.2016 ;41(4), 286-289.

19. Tebere H, Moifo B, Fongang $\mathrm{E}$ et al .Anévrisme géant de l'artère pulmonaire :a propos d'un cas .J Mal Vasc.2013;38(3) :201-205.

20. Hiroshi Y ,Fumio Y , Fuminobu T et al. Anévrisme d'une aorte thoracique descendante droite avec crosse à gauche et artère sous Clavière droite aberrante. Ann chirg vasc.2010;24(6) :896.e1-896 e5.

21. Mleyhi S, Ghedira F, Garali B et al. Anévrisme de l'aorte abdominale sous rénale rompu inaugurale d'une maladie de Takayasu chez un homme de 39 ans. J Mal Vasc. 2013 ;38(6) :373-376.

22. Daldoul S , Eddine KA, Khmili H et al. Rupture spontanée d'un anévrisme de l'artère mésentérique supérieure :a propos de deux cas . J Mal Vasc. $2014 ; 39(1)$ :62-66 